\title{
FIXED POINTS OF NONEXPANSIVE MAPPINGS IN BANACH LATTICES
}

\author{
M. A. KHAMSI AND PH. TURPIN
}

(Communicated by William J. Davis)

\begin{abstract}
We prove the existence of a fixed point for a nonexpansive mapping operating in a convex subset of a Banach lattice $E$ compact for some natural topology $\tau$ on $E$. In particular, if $E$ is a Banach space with a 1-unconditional basis we can take for $\tau$ the topology of coordinatewise convergence.
\end{abstract}

\section{INTRODUCTION}

If $B$ is a subset of a Banach space, a map $T: B \rightarrow B$ is said to be nonexpansive when the inequality

$$
\|T(x)-T(y)\| \leq\|x-y\|
$$

holds for every pair $x, y$ in $B$.

The main result of this paper (Corollary 1) is the following one. Let $E$ be a Banach space endowed with a 1-unconditional Schauder basis, i.e. a Schauder basis $\left(e_{n}\right)_{n \geq 0}$ such that $\left\|x_{0} e_{0}+\cdots+x_{n} e_{n}+\cdots\right\| \leq\left\|y_{0} e_{0}+\cdots+y_{n} e_{n}+\cdots\right\|$ if $\left|x_{n}\right| \leq\left|y_{n}\right|$ for every $n$ (in fact, this condition will be slightly weakened). Let $B$ be a convex nonvoid subset of $E$, compact for the topology of coordinatewise convergence. Then every nonexpansive map $T: B \rightarrow B$ has a fixed point.

This was proved by $\mathrm{Lin}[\mathrm{Ln}]$ in the special case where $B$ is a weakly compact convex set. The method of $[\mathrm{Ln}]$ is a refinement of techniques of Maurey [M, E-L-O-S]. It turns out that this method still works with the topology of coordinatewise convergence once a key lemma of Goebel and Karlovitz [G, Ka] has been generalized (Lemmas 4 and 5). Let us notice that our proof avoids any use of ultraproducts.

In fact, in the theorem below, we give a more general result, considering arbitrary Banach lattices $E$ and proving the above fixed point property in convex subsets compact for some natural topology $\tau$ on $E$. In usual spaces of measurable functions with order continuous norm (Corollary 2$), \tau$ is the topology of convergence in measure on every set with finite measure.

Received by the editors September 3, 1987 and, in revised form, January 21, 1988.

1980 Mathematics Subject Classification (1985 Revision). Primary 47H10; Secondary 46B30, 46B05. 
Related results can be found in [B-S] for weakly compact convex subsets of Banach lattices and in [LD-T] for $\tau$-compact star-shaped subsets of Orlicz spaces. The techniques of [LD-T], using the unicity of "asymptotic centers", exclude such spaces as $c_{0}$. In [M, E-L-O-S, B-S] the norm must be in some sense far from that of $l_{1}$. The method of [Ln], which is generalized here, avoids these restrictions.

A survey on fixed points and nonexpansive mappings is given in [Ki2].

Let us recall that Alspach [A] constructed a weakly compact convex subset of $L_{1}(0,1)$ invariant under some nonexpansive map without fixed point.

\section{THE RESULTS}

Theorem. Let $(E,\|\cdot\|)$ be a real Banach space, endowed with a vector lattice structure satisfying

$$
\left(x^{+} \leq y^{+} \text {and } x^{-} \leq y^{-}\right) \Rightarrow\|x\| \leq\|y\|, \quad x, y \text { in } E,
$$

and, for some real constant $k<2$,

$$
|x| \leq|y| \Rightarrow\|x\| \leq k\|y\|, \quad x, y \text { in } E .
$$

Let $\tau$ be the coarsest linear topology on $E$ for which the map $x \mapsto\||x| \wedge u\|$ from $E$ to $\mathbf{R}^{+}$is continuous at 0 for every $u \in E^{+}$.

Let $B$ be a $\tau$-compact nonvoid convex subset of $E$.

Then every nonexpansive map $T: B \rightarrow B$ has a fixed point.

The above topology $\tau$ may also be defined in the following way: it admits as a basis of neighborhoods of a point $x \in E$ the sets

$$
\{y \in E:\||y-x| \wedge u\| \leq \varepsilon\}, \quad u \in E^{+}, \varepsilon>0 .
$$

For example let $E$ be a real Banach space endowed with an unconditional Schauder basis $\left(e_{n}\right)_{n \geq 0}$. Then $E$ is a vector lattice for the "coordinatewise order", defined by $\sum_{n} x_{n} e_{n} \leq \sum_{n} y_{n} e_{n}$ when $x_{n} \leq y_{n}$ for every $n \geq 0$. The topology $\tau$ is easily seen to be the topology of coordinatewise convergence. Putting $x=\sum_{n=0}^{\infty} x_{n} e_{n}$ the conditions $(\alpha)$ and $(\beta)$ are respectively equivalent to

$$
\begin{aligned}
& \left\|\sum_{n=0}^{\infty} \varepsilon_{n} x_{n} e_{n}\right\| \leq\|x\|, \quad \varepsilon_{n}=0,1, x \in E, \\
& \left\|\sum_{n=0}^{\infty} \varepsilon_{n} x_{n} e_{n}\right\| \leq k\|x\|, \quad \varepsilon_{n}= \pm 1, x \in E .
\end{aligned}
$$

For instance, $\left(\alpha^{\prime}\right)$ implies $(\alpha)$ because, if $0 \leq t_{n} \leq 1$ for every $n$, $\sum_{0}^{\infty} t_{n} x_{n} e_{n}$ lies in the closed convex hull of the points $\sum_{0}^{\infty} \varepsilon_{n} x_{n} e_{n}, \varepsilon_{n}=0,1$.

So we get the following special case of the theorem. 
Corollary 1. Let $E$ be a real Banach space endowed with an unconditional Schauder basis satisfying the above conditions $\left(\alpha^{\prime}\right)$ and $\left(\beta^{\prime}\right)$, with $k<2$. Let $B$ be a nonvoid convex subset of $E$, compact for the topology of coordinatewise convergence.

Then every nonexpansive map $T: B \rightarrow B$ has a fixed point.

When $B$ is weakly compact, the above statement is due to $\operatorname{Lin}[\mathrm{Ln}]$.

Example 1. Let $B$ be a nonvoid convex subset of the space $l_{1}(\mathbf{N})$ of absolutely summable sequences, weak ${ }^{*}$-compact for the usual duality with $c_{0}(\mathbf{N})$ (or, equivalently, coordinatewise compact for the canonical basis $\left(e_{n}\right)$ of $\left.l_{1}(\mathbf{N})\right)$. Then $B$ has a fixed point for a mapping $T: B \rightarrow B$ if $T$ is nonexpansive for the norm

$$
p_{\lambda}(x)=\|x\|_{1} \vee\left(\lambda\|x\|_{\infty}\right)
$$

where $\lambda$ is some real number, $\left\|\left(x_{n}\right)\right\|_{1}=\sum_{0}^{\infty}\left|x_{n}\right|,\left\|\left(x_{n}\right)\right\|_{\infty}=\sup _{n}\left|x_{n}\right|$.

This result is evident for the weakly compact convex sets since these sets are in fact compact in norm, but we see no obvious way to deduce it from known results for arbitrary weak ${ }^{*}$-compact convex sets if $\lambda \geq 2$.

For instance, let us consider the weak ${ }^{*}$-compact convex set $B=\{x \in$ $\left.l_{1}^{+}:\|x\|_{1} \leq 1\right\}$. It contains the unit vectors $e_{n}, n \geq 0$. If $\lambda \geq 2$, then, for every $x \in B, p_{\lambda}\left(x-e_{n}\right)$ tends to the diameter $\lambda$ of $B$ as $n \rightarrow \infty$. This shows that $B$ has no weak ${ }^{*}$-normal structure [Ki2] if $\lambda \geq 2$. So the methods using normal structures cannot be applied in this case.

On the other hand, in [B-S], Borwein and Sims generalize the technique of [M] to some Banach lattices $E$ with a "Riesz angle" $\alpha(E)=\sup \{\||x| \vee|y|\|:\|x\| \vee$ $\|y\| \leq 1\}$ satisfying $\alpha(E)<2$. The Riesz angle of $\left(l_{1}, p_{\lambda}\right)$ is equal to 2 , so the space $\left(l_{1}, p_{\lambda}\right)$ does not fall under the scope of [B-S]. Moreover only weakly compact convex sets are considered in [B-S]. However, let us consider the Banach lattice $c(\mathbf{N})$ of convergent sequences, endowed with the supremum norm. It is proved in [B-S] that every weakly compact convex subset of $c(\mathbf{N})$ has the "nonexpansive fixed point property." This is not given by our theorem. Indeed the topology $\tau$ of $c(\mathbf{N})$ is the norm-topology.

Let us observe that the conclusion of Corollary 1 is false for $k=2$, as shown by an example of $\mathrm{Lim}$ [Lm, Ln].

Corollary 1 is concerned with sequence spaces. It can be generalized to spaces of measurable functions in the following way.

Let $(\Omega, \mathscr{A}, \mu)$ be a measure space and let $M=M(\Omega, \mathscr{A}, \mu)$ be the vector lattice of all $\mu$-classes of $\mu$-measurable functions on $(\Omega, \mathscr{A}, \mu)$. An order ideal of $M$ is a vector subspace $E$ of $M$ such that $x \in E$ as soon as $x \in M$ and $|x| \leq|y|$ for some element $y$ of $E$. A norm $\|\cdot\|$ on $E$ is said to be sequentially order continuous when $\lim _{n}\left\|x_{n}\right\|=0$ for every decreasing sequence $\left(x_{n}\right)$ of $E^{+}$with $\inf _{n} x_{n}=0$.

Corollary 2. Let $E$ be an order ideal of $M(\Omega, \mathscr{A}, \mu)$ and a Banach space for a sequentially order continuous norm satisfying conditions $(\alpha)$ and $(\beta)$ of the 
theorem, for some constant $k<2$. We assume also that an element $x$ of $E$ is null provided $x=0$ a.e. on every set $A \in \mathscr{A}$ with finite measure. Let $B$ be a nonvoid convex subset of $E$, compact for the topology $\sigma$ of convergence in measure on every set $A \in \mathscr{A}$ with finite measure. Then every nonexpansive map $T: B \rightarrow B$ has a fixed point.

This topology $\sigma$ on $E$ admits as a basis of neighborhoods of a point $x_{0} \in E$ the family of sets

$$
V\left(x_{0}, A, a, \varepsilon\right)=\left\{x \in E: \mu\left(A \cap\left(\left|x-x_{0}\right|>a\right)\right)<\varepsilon\right\},
$$

where $a>0, \varepsilon>0$ and $A \in \mathscr{A}$ with $\mu(A)<\infty$. When $\mu$ is the counting measure on the power set $2^{\Gamma}$ of a nonvoid set $\Gamma, \sigma$ is the topology of pointwise convergence.

Proof of Corollary 2. It suffices to check that $\sigma$ is finer than $\tau$, i.e. that the mapping $x \rightarrow\||x| \wedge u\|$ from $(E, \sigma)$ to $\mathbf{R}^{+}$is continuous at 0 for every $u \in E^{+}$, and to apply the theorem. So, let $u \in E^{+}, u \neq 0$, and let $\left(A_{i}\right)_{i \in I}$ be a maximal disjoint family of sets $A_{i} \in \mathscr{A}$ such that $\mu\left(A_{i}\right)<\infty$ and $\left\|u 1_{A_{i}}\right\|>0$, where $1_{A_{i}}$ is the characteristic function of $A_{i}$. Using the sequential order continuity of the norm, it is easily checked that $I$ is finite or countable. Applying again this property, one can find a set $A \in \mathscr{A}$ for which $\mu(A)<\infty$ and $\left\|u-u 1_{A}\right\|<\varepsilon$, where $\varepsilon>0$ is given. One can also find real numbers $a>0, b>0, \eta>0$ satisfying $\left\|u 1_{(u<a)}\right\|<\varepsilon,\|b u\|<\varepsilon$ and $\left\|u 1_{B}\right\|<\varepsilon$ for any $B \in \mathscr{A}$ with $\mu(B)<\eta$. Then, if $x \in E$ and $\mu(A \cap(|x|>a b))<\eta$, we get (using condition $(\alpha))\||x| \wedge u\| \leq\left\|u-u 1_{A}\right\|+\left\|u 1_{(u<a)}\right\|+\|b u\|+\left\|u 1_{A \cap(|x|>a b)}\right\| \leq 4 \varepsilon$.

Example 2. Let $L_{\phi}=L_{\phi}(\Omega, \mathscr{A}, \mu)$ be an Orlicz space, $\phi$ being a convex Orlicz function (see [L-T]). In Corollary 2 we can take for $E$ the closed linear subspace $L_{\phi}^{0}$ of $L_{\phi}$ generated by the $\mu$-integrable simple functions, endowed with any Riesz norm equivalent to the usual Luxemburg norm (in this case the topologies $\sigma$ and $\tau$ on $E$ are identical).

Let us observe [LD-T, Theorem 10.1] that if $\phi$ verifies the condition $\Delta_{2}$ (i.e. if $L_{\phi}^{0}=L_{\phi}$ ) and if $E=L_{\phi}$ is endowed with the Luxemburg norm, the set $B$ of Corollary 2 need not be convex: it suffices to suppose $B$ star-shaped, bounded in norm and compact for the topology $\sigma$.

\section{Proof of THE THEOREM}

The hypotheses are those of the theorem.

\section{Lemma 1.}

(a) The topology $\tau$ is a Hausdorff linear topology, coarser than the topology defined by the norm.

(b) Every convex $\tau$-compact subset $C$ of $E$ is norm-bounded.

(c) If $K$ is a $\tau$-compact subset of $E$, every sequence $\left(x_{n}\right)$ of points of $K$ contains a $\tau$-convergent subsequence. 
Proof of (a). Obvious.

Proof of (b). When $C$ is nonvoid, let $E_{C}$ be the vector subspace of $E$ generated by $C$. Clearly, the functional $\|x\|_{C}=\inf \{t>0: x \in t(C-C)\}$, $x \in E_{C}$, is a norm on $E_{C}$, the canonical injection of $\left(E_{C},\|\cdot\|_{C}\right)$ into $(E, \tau)$ is continuous, and $\left(E_{C},\|\cdot\|_{C}\right)$ is a Banach space: if $\left(x_{n}\right) \subset C-C$ is a Cauchy sequence for $\|\cdot\|_{C}$ and $x$ is a $\tau$-cluster point of $\left(x_{n}\right)$, it is easily seen that $\left\|x-x_{n}\right\|_{C}$ tends to 0 . It remains to apply the closed graph theorem to the canonical injection of $\left(E_{C},\|\cdot\|_{C}\right)$ into $(E,\|\cdot\|)$.

Proof of $(\mathrm{c})$. Let $L$ be the closure in $(E, \tau)$ of the set of the elements $y$ of $E$ satisfying $|y| \leq N \sum_{0}^{N}\left|x_{n}\right|$ for some integer $N$ ( $L$ is the $\tau$-closed order ideal of $E$ generated by the $x_{n}$ 's). Let $\tau_{L}$ be the linear topology on $L$ which admits as a basis of zero-neighborhoods the sets

$$
V_{N}(\varepsilon)=\left\{x \in L:\left\||x| \wedge \sum_{n=0}^{N}\left|x_{n}\right|\right\| \leq \varepsilon\right\}, \quad \varepsilon>0, N=0,1, \ldots
$$

This topology $\tau_{L}$ on $L$ is coarser than the topology induced by $\tau$. Let us prove that $\tau_{L}$ is Hausdorff (then $\tau_{L}$ is metrizable, $\tau$ and $\tau_{L}$ coincide on $K \cap L$ by compactness, and we are done). We have to show that $x$ is null if $x \in L$ and $|x| \wedge \sum_{0}^{N}\left|x_{n}\right|=0$ for every $N$. But $\{y \in E:|x| \wedge|y|=0\}$ is a $\tau$-closed order ideal of $E$ (it is easily checked that the lattice operations of $E$ are $\tau$-continuous), so it contains $x$, whence $x=0$.

Lemma 2. Let $\left(u_{n}\right)$ and $\left(v_{n}\right)$ be sequences of $E$ converging to some point $c \in E$ for the topology $\tau$, with $\lim _{n}\left\|\left|u_{n}-c\right| \wedge\left|v_{n}-c\right|\right\|=0$. Then, for every sequence $\left(w_{n}\right)$ of $E$ and for every $x \in E$, we have

$$
\begin{aligned}
2 \lim \sup _{n}\left\|w_{n}-c\right\| \leq & \lim \sup _{n}\left\|w_{n}-x\right\|+\limsup _{n}\left\|w_{n}-u_{n}\right\| \\
& +\lim \sup _{n}\left\|w_{n}-v_{n}\right\| .
\end{aligned}
$$

Proof. For $u$ and $x$ in $E$ let

$$
S_{u}(x)=x^{+} \wedge|u|-x^{-} \wedge|u| .
$$

Then, for $u, v, x, y$ in $E$ the following inequalities hold.

$$
\begin{aligned}
\left\|S_{u}(x)\right\| & \leq\|x\| \wedge(k\|u\|), \\
\left\|x-S_{u}(x)\right\| & \leq\|x-u\|, \\
\left\|S_{u}(x)-S_{u}(y)\right\| & \leq 2 k\||x-y| \wedge|u|\|, \\
\left\|S_{u}(x)+S_{v}(x)\right\| & \leq\|x\|+k\||u| \wedge|v|\| .
\end{aligned}
$$

We get (1) using $(\alpha)$ and $(\beta)$ since $\left(S_{u}(x)\right)^{+} \leq x^{+},\left(S_{u}(x)\right)^{-} \leq x^{-}$and $\left|S_{u}(x)\right| \leq|u|$. We also have $\left(x-S_{u}(x)\right)^{+}=(x-|u|)^{+} \leq(x-u)^{+}$and, changing $x$ and $u$ into their opposites, $\left(x-S_{u}(x)\right)^{-} \leq(x-u)^{-}$; this gives (2). The inequality (3) is given by

$$
\begin{aligned}
\left|S_{u}(x)-S_{u}(y)\right| & \leq\left|x^{+} \wedge\right| u\left|-y^{+} \wedge\right| u||+\left|y^{-} \wedge\right| u\left|-x^{-} \wedge\right| u|| \\
& \leq\left|x^{+}-y^{+}\right| \wedge|u|+\left|y^{-}-x^{-}\right| \wedge|u| \leq 2(|x-y| \wedge|u|) .
\end{aligned}
$$


We get (4) using (1) and the equality $S_{u}(x)+S_{v}(x)=S_{|u| \vee|v|}(x)+S_{|u| \wedge|v|}(x)$, an easy consequence of the identity $a+b=a \vee b+a \wedge b$.

Now we prove Lemma 2 as follows. Without loss of generality, we assume $c=0$. Using (2) we have

$$
\begin{aligned}
2\left\|w_{n}\right\| & \leq\left\|S_{u_{n}}\left(w_{n}\right)+S_{v_{n}}\left(w_{n}\right)\right\|+\left\|w_{n}-S_{u_{n}}\left(w_{n}\right)\right\|+\left\|w_{n}-S_{v_{n}}\left(w_{n}\right)\right\| \\
& \leq\left\|S_{u_{n}}\left(w_{n}\right)+S_{v_{n}}\left(w_{n}\right)\right\|+\left\|w_{n}-u_{n}\right\|+\left\|w_{n}-v_{n}\right\| .
\end{aligned}
$$

By (3) and since $\lim _{n} u_{n}=0$ for the topology $\tau$, we have

$$
\limsup _{n}\left\|S_{u_{n}}\left(w_{n}\right)-S_{u_{n}}\left(w_{n}-x\right)\right\| \leq 2 k \lim \sup _{n}\left\||x| \wedge\left|u_{n}\right|\right\|=0,
$$

and similarly $\left\|S_{v_{n}}\left(w_{n}\right)-S_{v_{n}}\left(w_{n}-x\right)\right\|$ tends to 0 . So, using (4)

$$
\begin{aligned}
& \operatorname{lim\operatorname {sup}}\left\|S_{u_{n}}\left(w_{n}\right)+S_{v_{n}}\left(w_{n}\right)\right\|=\limsup _{n}\left\|S_{u_{n}}\left(w_{n}-x\right)+S_{v_{n}}\left(w_{n}-x\right)\right\| \\
& \leq \limsup \left\|w_{n}-x\right\|+k \lim \sup _{n}\left\|\left|u_{n}\right| \wedge\left|v_{n}\right|\right\|=\underset{n}{\lim \sup }\left\|w_{n}-x\right\| .
\end{aligned}
$$

This achieves the proof of Lemma 2.

A sequence $\left(z_{n}\right)$ of $B$ is said to be an "approximate fixed points sequence" when it verifies

$$
\lim _{n}\left\|T\left(z_{n}\right)-z_{n}\right\|=0 \text {. }
$$

Lemma 3. There exists in $B$ a point $c$ and an approximate fixed points sequence $\left(z_{n}\right)$ satisfying

$$
\limsup _{n}\left\|z_{n}-c\right\| \leq \frac{1}{4}(k+2) \operatorname{Diam}(B),
$$

where $\operatorname{Diam}(B)=\sup \{\|x-y\|:(x, y) \in B \times B\}$.

Proof. For $u \in B$ and $\lambda \in(0,1)$ the mapping

$$
T_{u, \lambda}(x)=(1-\lambda) u+\lambda T(x), \quad x \in B,
$$

satisfies $\left\|T_{u, \lambda}(x)-T_{u, \lambda}(y)\right\| \leq \lambda\|x-y\|$ for $x, y$ in $B$. So it has a unique fixed point $u(\lambda) \in B$. We have for $u, v$ in $B$ and $\lambda \in(0,1)$

$$
\begin{aligned}
& T(u(\lambda))=\left(1-\lambda^{-1}\right) u+\lambda^{-1} u(\lambda), \\
& \|u(\lambda)-u\| \leq \lambda(1-\lambda)^{-1}\|T(u)-u\|, \\
& \|u(\lambda)-v(\lambda)\| \leq\|u-v\|
\end{aligned}
$$

The equality (7) is obvious. Since $T_{u, \lambda}$ is $\lambda$-Lipschitzian and $T_{u, \lambda}(u(\lambda))=$ $u(\lambda)$, we have clearly $\|u(\lambda)-x\| \leq(1-\lambda)^{-1}\left\|T_{u, \lambda}(x)-x\right\|$ for every $x \in B$, whence (8) taking $x=u$. Finally, we deduce (9) from

$$
\begin{aligned}
\| u(\lambda)-v(\lambda \| & =(1-\lambda)(u-v)+\lambda(T(u(\lambda))-T(v(\lambda)) \| \\
& \leq(1-\lambda)\|u-v\|+\lambda \dot{\|} u(\lambda)-v(\lambda) \| .
\end{aligned}
$$

As is well known, the $u(\lambda)$ 's yield approximate fixed points sequences since $\|T(u(\lambda))-u(\lambda)\|=\|(1-\lambda)(T(u(\lambda))-u)\| \leq(1-\lambda) \operatorname{Diam}(B)$, with $\operatorname{Diam}(B)<\infty$ 
(Lemma 1(b)). So, using Lemma 1(c), we can find in $B$ an approximate fixed points sequence $\left(c_{n}\right)_{n \geq 0} \tau$-converging to some point $c \in B$. By definition of $\tau$, we may even assume that $\left\|\left|c_{2 n}-c\right| \wedge\left|c_{2 n+1}-c\right|\right\|$ tends to 0 . Hence, letting $u_{n}=c_{2 n}, v_{n}=c_{2 n+1}$, we have

$$
\begin{gathered}
\lim _{n}\left\|T\left(u_{n}\right)-u_{n}\right\|=\lim _{n}\left\|T\left(v_{n}\right)-v_{n}\right\|=0, \\
\tau-\lim _{n} u_{n}=\tau-\lim _{n} v_{n}=c, \\
\lim _{n}\left\|\left|u_{n}-c\right| \wedge\left|v_{n}-c\right|\right\|=0 .
\end{gathered}
$$

Then the sequence $w_{n}=\frac{1}{2}\left(u_{n}+v_{n}\right), n=0,1, \ldots$, satisfies

$$
\limsup _{n}\left\|w_{n}-c\right\| \leq \frac{k}{2} \operatorname{Diam}(B) .
$$

Indeed, for $x, y$ in $E$ we have

$$
\begin{aligned}
\|x+y\| & \leq\left\|x+y-2\left(x^{+} \wedge y^{+}\right)+2\left(x^{-} \wedge y^{-}\right)\right\|+2\left\|x^{+} \wedge y^{+}-x^{-} \wedge y^{-}\right\| \\
& \leq k\|x-y\|+2 k\||x| \wedge|y|\|,
\end{aligned}
$$

since $\left|x^{+} \wedge y^{+}-x^{-} \wedge y^{-}\right| \leq|x| \wedge|y|$ and the identity $a+b-2(a \wedge b)=|a-b|$ gives $\left|x+y-2\left(x^{+} \wedge y^{+}\right)+2\left(x^{-} \wedge y^{-}\right)\right|=|| x^{+}-y^{+}|-| x^{-}-y^{-}|| \leq|x-y|$. Applying this with $x=u_{n}-c$ and $y=v_{n}-c$ and using (12) we get (13).

Let us now pick some fixed number $\lambda \in(0,1)$. From (9) and (13) we deduce

$$
\limsup _{n}\left\|w_{n}(\lambda)-c(\lambda)\right\| \leq \frac{k}{2} \operatorname{Diam}(B) .
$$

Furthermore we have

$$
\limsup _{n}\left(\left\|w_{n}(\lambda)-u_{n}\right\| \vee\left\|w_{n}(\lambda)-v_{n}\right\|\right) \leq \frac{1}{2} \operatorname{Diam}(B)
$$

Indeed, by (8) and (10), $\lim _{n}\left\|u_{n}(\lambda)-u_{n}\right\|=0$, so using (9),

$$
\limsup _{n}\left\|w_{n}(\lambda)-u_{n}\right\| \leq \limsup _{n}\left\|w_{n}-u_{n}\right\| \leq \frac{1}{2} \operatorname{Diam}(B) \text {. }
$$

The same is true for $\left\|w_{n}(\lambda)-v_{n}\right\|$, whence (15). In view of (11), (12), (14) and (15), Lemma 2 gives

$$
\limsup _{n}\left\|w_{n}(\lambda)-c\right\| \leq \frac{1}{4}(k+2) \operatorname{Diam}(B) .
$$

Now, if $\lambda_{n} \in(0,1), n=0,1, \ldots$, with $\lim _{n} \lambda_{n}=1$, there exists a sequence $z_{n}=w_{h_{n}}\left(\lambda_{n}\right)$ such that $\lim \sup _{n}\left\|z_{n}-c\right\| \leq \frac{1}{4}(k+2) \operatorname{Diam}(B)$. By $(7),\left(z_{n}\right)$ is an approximate fixed points sequence: this proves Lemma 3.

The following lemma is essentially due to Goebel and Karlovitz [G, Ka]. In [ $\mathrm{G}$ and $\mathrm{Ka}$ ] the topology $\alpha$ below is the weak topology.

Lemma 4 (Goebel, Karlovitz). Let $F$ be a Banach space and let $K$ be a convex bounded nonvoid subset of $F$, compact for some Hausdorff topology $\alpha$. We assume that, for every sequence $\left(x_{n}\right)$ of $K$, the map $r(c)=\lim \sup _{n}\left\|x_{n}-c\right\|$, $c \in K$, is lower semicontinuous on $K$ for the topology $\alpha$. Let $T: K \rightarrow K$ be a 
nonexpansive mapping. We suppose that $K$ is minimal, in the sense that every $\alpha$-compact convex nonvoid $T$-invariant subset of $K$ is equal to $K$.

Then we have

$$
\lim _{n}\left\|x_{n}-c\right\|=\operatorname{Diam}(K)
$$

for every approximate fixed points sequence $\left(x_{n}\right)$ of $K$ and every $c \in K$.

As the above mapping $r(c)$ is weakly lower semicontinuous (it is convex and norm-continuous), Lemma 4 contains the statement of Goebel and Karlovitz.

Proof. First we prove that we have, for every $x \in K$,

$$
\sup \{\|x-c\|: c \in K\}=\operatorname{Diam}(K) .
$$

When $\alpha$ is the weak topology this is a result of Kirk [Kil], and we follow Kirk's proof. Of course the hypothesis on $\alpha$ implies that the map $x \rightarrow\|x-c\|$, $x \in K$, is $\alpha$-l.s.c. for every $c \in K$; hence, so is the map $d(x)=\sup _{c \in K}\|x-c\|$. Therefore, if $m=\inf _{x \in K} d(x)$, the set $K_{0}=\{x \in K: d(x)=m\}$ is a nonvoid $\alpha$-compact convex subset of $K$. It remains to prove that $K_{0}$ is $T$-invariant (then $K_{0}=K$ and $\left.m=\operatorname{Diam}(K)\right)$. So, let $x \in K_{0}$. The set $\{c \in K: \| T(x)-$ $c \| \leq m\}$ is an $\alpha$-compact convex set which is nonvoid and $T$-invariant since it contains $T(K)$ by nonexpansiveness of $T$. Hence it is equal to $K$ and $T(x) \in K_{0}$.

As a consequence of (16) we get $\sup \{r(c): c \in K\}=\operatorname{Diam}(K)$ for every sequence $\left(x_{n}\right)$ of $K$ if $r(c)=\limsup \left\|x_{n}-c\right\|$ : indeed $r(c) \geq\|x-c\|$ if $x$ is an $\alpha$-cluster point of $\left(x_{n}\right)$, by $\alpha$-lower semicontinuity ( $\alpha$-l.s.c.). But if $\left(x_{n}\right)$ is an approximate fixed points sequence, the map $r(c)$ is convex, $\alpha$-l.s.c. and verifies $r(T(c)) \leq r(c)$. By minimality of $K$, it is constant on $K$. So $r(c)=\operatorname{Diam}(K)$ for every $c \in K$, which easily gives the lemma.

The following result shows that Lemma 4 can be applied to the space $E$ of the theorem, with $\alpha=\tau$.

Lemma 5. For every norm-bounded and relatively $\tau$-compact sequence $\left(x_{n}\right)$ of $E$, the mapping $r(c)=\limsup _{n}\left\|x_{n}-c\right\|, c \in E$, is lower semicontinuous on $(E, \tau)$.

Proof. Let $c \in E$ and $\varepsilon>0$. It suffices to show that $r(c+x) \geq r(c)-\varepsilon$ for every $x$ in some $\tau$-neighborhood $V$ of the origin. Using Lemma 1 we have $r(c)=\lim _{n}\left\|y_{n}\right\|$ for some subsequence $\left(y_{n}\right)$ of $\left(x_{n}-c\right)$ converging to some point $y \in E$ for the topology $\tau$. The set $V=\left\{x \in E:\||y| \wedge|x|\| \leq k^{-1} \varepsilon / 3\right\}$ is a $\tau$-neighborhood of the origin. Let $x \in V$. Since $\lim _{n}\left\|\left|y_{n}-y\right| \wedge|x|\right\|=0$ we have

$$
\limsup \left\|\left|y_{n}\right| \wedge|x|\right\| \leq k^{-1} \varepsilon / 3 \text {. }
$$

Using the functions $S_{u}$ (cf. Lemma 2) this gives

$$
\limsup _{n}\left\|y_{n}-x\right\| \geq \limsup _{n}\left\|y_{n}-S_{x}\left(y_{n}\right)-\left(x-S_{y_{n}}(x)\right)\right\|-2 \varepsilon / 3
$$


since $\left|S_{x}\left(y_{n}\right)\right|=\left|S_{y_{n}}(x)\right|=\left|y_{n}\right| \wedge|x|$. But $y_{n}-S_{x}\left(y_{n}\right)$ and $x-S_{y_{n}}(x)$ are easily seen to be disjoint. So (condition $(\alpha)$ of the theorem), we get

$$
\begin{aligned}
\limsup _{n}\left\|y_{n}-x\right\| & \geq \limsup _{n}\left\|y_{n}-S_{x}\left(y_{n}\right)\right\|-2 \varepsilon / 3 \\
& \geq \lim _{n}\left\|y_{n}\right\|-\varepsilon
\end{aligned}
$$

whence $r(c+x) \geq r(c)-\varepsilon$.

Proof of the theorem. Using Zorn's lemma we may assume that the set $B$ of the theorem is minimal (cf. Lemma 4). Let us consider the approximate fixed points sequence $\left(z_{n}\right) \subset B$ and the point $c \in B$ given by Lemma 3 . In view of Lemmas 4 and 5 (and Lemma $1(b)$ ) we have $\lim _{n}\left\|z_{n}-c\right\|=\operatorname{Diam}(B)$, whence $\operatorname{Diam}(B)=0$ if $k<2$.

\section{REFERENCES}

[A] D. Alspach, A fixed point free nonexpansive map, Proc. Amer. Math. Soc. 82 (1981), 423-424.

[B-S] J. M. Borwein and B. Sims, Non-expansive mappings on Banach lattices and related topics, Houston J. of Math. 10 (1984), 339-356.

[E-L-O-S] J. Elton, P. K. Lin, E. Odell and S. Szarek, Remarks on the fixed point problem for nonexpansive maps, Contemp. Math., vol. 18, Amer. Math. Soc., Providence, R.I., 1983, pp. 87-120.

[G] K. Goebel, On the structure of minimal invariant sets for nonexpansive mappings, Ann. Univ. Mariae Curie-Skłodowska 29 (1975), 73-77.

[Ka] L. A. Karlovitz, Existence of fixed points of nonexpansive mappings in a space without normal structure, Pacific J. Math. 66 (1976), 153-159.

[Ki1] W. A. Kirk, A fixed point theorem for mappings which do not increase distances, Amer. Math. Monthly 72 (1965), 1004-1006.

[Ki2] __ Fixed point theory for nonexpansive mappings. II, Contemp. Math., vol. 18, Amer. Math. Soc., Providence, R.I., 1983, pp. 121-140.

[LD-T] E. Lami-Dozo and Ph. Turpin, Nonexpansive maps in generalized Orlicz spaces, Studia Math. 86 (1987), 155-188.

[Lm] T. C. Lim, Asymptotic centers and nonexpansive mappings in conjugate Banach spaces, Pacific J. Math. 90 (1980), 135-143.

[Ln] P. K. Lin, Unconditional bases and fuxed points of nonexpansive mappings, Pacific J. Math. 116 (1985), 69-76.

[L-T] J. Lindenstrauss and L. Tzafriri, Classical Banach spaces. II, Springer-Verlag, Berlin, Heidelberg and New York, 1979.

[M] B. Maurey, Points fixes des contractions de certains faiblement compacts de $L^{1}$, Séminaire d'Analyse Fonctionnelle 1980-81, Exposé N ${ }^{\circ}$ VIII, Ecole Polytechnique, Palaiseau, 1981.

Equipe d'Analyse, Equipe de Recherche associée au C.N.R.S. N` 294, Université Paris VI, 4, Place Jussieu, Tour 46, 4kme étage, 75230 Paris Cedex 05, France

Université Paris XI, Mathématique (BÂt. 425), 91405 Orsay, France 